Revue d'histoire de l'Amérique française

Q6. REVUE D.HISTOIRE DE L'AMÉRIQUE FRANÇAISE

\title{
Table des auteurs de compte rendus
}

Volume 30, numéro 4, index, mars 1977

Index des volumes XXI-XXX (juin 1967-mars 1977)

URI : https://id.erudit.org/iderudit/303575ar

DOI : https://doi.org/10.7202/303575ar

Aller au sommaire du numéro

Éditeur(s)

Institut d'histoire de l'Amérique française

ISSN

0035-2357 (imprimé)

1492-1383 (numérique)

Découvrir la revue

Citer ce document

(1977). Table des auteurs de compte rendus. Revue d'histoire de l'Amérique

française, 30(4), 99-118. https://doi.org/10.7202/303575ar d'utilisation que vous pouvez consulter en ligne.

https://apropos.erudit.org/fr/usagers/politique-dutilisation/ 
TABLE

DES

AUTEURS DE

COMPTES

RENDUS 
Allard, Michel: Canadian Jackdaws, 23,3: 482-484. - Dubé, Jean-Claude, Claude-Thomas Dupuy, intendant de la Nouvelle-France 1678-1738, 23,2: 334-335. - Farley-Lamarche/Boréal Express, Histoire 1534-1968, 22,3: 472-474. - Hodgetts, A. B., Quelle culture? Quel héritage?, 22,4: 643-647. - Lefebvre, André, Le Jeu de la Vie, 24,1: 99-101. - Le Professeur d'histoire, no 1, 22,3: 476-477.

Allen, Patrick: Rumilly, Robert, Histoire de l'École des Hautes Études Commerciales de Montréal, 21,1: 149-151.

Angers, François-Albert: TurcotteGirard, Judith, La paroisse aux trois églises, 27,3: 441-442.

Arès, Richard, s.j.: Beck, J. M., The Shaping of Canadian Federalism: Central Authority or Provincial Right?, n.c., 28,2: 245-246. - Bouthillier, Guy et Jean Meynaud, Le choc des langues au Québec, 1760-1970, 27,1: 93-95. - Burns, R. M., One country or Two? n.c., 28,2: 248-249. - Comité spécial mixte du Sénat et de la Chambre des Communes sur la Constitution du Canada. Rapport final, Le, n.c., 28,2: 250-252. - Falch, Jean, Contribution à l'étude du statut des langues en Europe, 28,2: 253. Faribault, Marcel, La révision constitutionnelle - Premiers fondements, 25,1: 117-119. - Gaboury, JeanPierre, Le Nationalisme de Lionel Groulx - Aspects idéologiques, 24,2: 287-290. - Groulx, Lionel, Mes Mémoires. Tome III: 1926-1939, 27,1: 105-106. - Hockin, Thomas, The Canadian Condominium. Domestic Issues and External Policy, 28,2: 249. Kloss, Heinz, Les droits linguistiques des Franco-Américains aux ÉtatsUnis, 25,3: 420-421. - Lalande, Gil- les, Pourquoi le fédéralisme, n.c., 28,2: 257-258. - Leeson, H. A. et W. Vanderelst, External Affairs and Canadian Federalism: The History of a Dilemma, n.c., 28,2: 246. - Morin, Claude, Le combat québécois, n.c., 28,2: 256. - Morin, Claude, Le pouvoir québécois... en négociation, n.c., 28,2: 255-256. - Prévost, Jean-Pierre, La crise du fédéralisme canadien, n.c., 28,2: 254-255. - Serbyn, Roman, Fédéralisme et nations, n.c., 28,2: 252253. - Simeon, Richard, FederalProvincial Diplomacy: The Making of recent Policy in Canada, n.c., 28,2: 246-247. - Smiley. Donald V., Canada in Question: Federalism in the Seventies, n.c., 28,2: 247-248. Thorson, J. T., Wanted: A Single Canada, n.c., 28,2: 249-250. - Touret, Bernard, L'aménagement constitutionnel des États de peuplement composite, n.c., 28,2: 253-254.

Armstrong, Frederick H.: Cooper, John Irwin, Montreal a Brief History, 23,4: 613-614.

Armstrong, Robert: Faucher, Albert, Québec en Amérique au XIXe siècle. Essai sur les caractères économiques de la Laurentie, 27,4: 586-590. - Nelles, H. V., The Politics of Development-Forest Mines \& HydroElectric Power in Ontario, 1849-1941, 29,4: 595-599.

Arthur, M. Elizabeth: Beaglehole, J. C., éd., The Journals of Captain James Cook - The Voyage of the Resolution and Discovery, 1776-1780, 21,4: 847849.

Audet, Louis-Philippe: Godbout, Arthur, L'origine des Ecoles françaises dans l'Ontario, 26,4: 588-590. - Jolois, Jean-Jacques, J.-F.-Perrault, 1753-1844 et les origines de l'enseignement laïque au Bas-Canada, 23,4: 618-625. 
Baillargeon, Georges: Hamer, Pierre, Raphaël de Luxembourg. Une contribution luxembourgeoise à la colonisation de la Louisiane, 22,1: 134-136.

Barros, Jacques: Pierre-Charles-Gérard, L'économie haïtienne et sa voie de développement, 22,1: 104-108.

Baudry, René: De Vaux De Foletier, François, Les Tsiganes dans l'Ancienne France, 21,2: 329-332. - Lee, Ronald, The Gypsies in Canada, 21,2: 329-332. - Meurgey de Tupigny, Jacques, Les Leriget, Leriget de la Faye, Leriget de la Plante... (Angoumois, Dauphiné... Canada...), 21,1: 147-148.

Beaudin, François: Nish, Cameron, Inventaire sommaire des archives de la Société historiqute de Montréa!, 23,3: 462-465.

Beaulieu, André: Les Cahiers des Dix, no $32,22,4: 647-650$.

Beauregard, Ludger: Morissonneau, Christian, La Société de géographie de Québec, 1877-1970, 26,1: 114-115.

Begin-Wolff, Claudette: Levitt, Joseph, Henri Bourassa and the Golden CalfThe Social Program of the Nationalists of Quebec, 1900-1914, 26,1: 111113.

Behiels, Michael: Renaud, Laurier, La fondation de l'A.C.J.C.; l'histoire d'une jeunesse nationaliste, 28,1: 119-120.

Bélanger, André.-J.: Gagnon, MarcelAimé, Jean-Charles Harvey, précurseur de la révolution tranquille, 26,2: 279-281.

Bernard, Jean-Paul: Costisella, Joseph, L'Esprit révolutionnaire dans la littérature canadienne-française à la fin du XIXe siècle, 23,2: 325-327. - Lefebvre, André, La Montreal Gazette et le nationalisme canadien (1835-1842), 27,4: 596-598. - Monet, Jacques, The Last Cannon Shot. A Study of French Canadian Nationalism, 1837-1850, 24,3: 435-437. - Ouellet, Fernand, Éléments d'histoire sociale du BasCanada, 27,2: 288-292. - Trudel, Marcel, Initiation à la NouvelleFrance, histoire et institutions, 22,1: 112-114. - Wallot, Jean-Pierre, Un Québec qui bougeait-Trame socio-po- litique du Québec au tournant du XIXe siècle, 29,3: 443-444.

Bernier, Benoît: Dictionnaire biographique du Canada, vol. X: 1871-1880, 27,1: 99-101: Falardeau, J.-C., «A propos d'Étienne Parent», Dictionnaire biographique du Canada, n.c., 27,1: 86-90. - Provost, Honorius, La Vallée de la Chaudière, géographie et histoire. Notes d'enseignement, 25,2: 261-262. - Richard, Joseph-Arthur, ptre, Histoire de Cap St-Ignace, 1672-1970, 25,3: 424-425.

Bertrand, Denis: Morin, Rosaire, L'Immigration au Canada, 23,4: 630-632. - Rumilly, Robert, La Guerre de 1939-1945, Ernest Lapointe, XXXVIIIe tome de l'Histoire de la province de Québec, 22,4: 633-636.

Bilodeau, Rosario: Archives publiques du Canada, (Coll.). Catalogue collectif des manuscrits des Archives canadiennes-Union List of Manuscripts in Canadian Repositories, 23,2: 327. - Archives publiques du Canada. Nouveaux documents sur Champlain et son époque (vol. I, 1560-1622), 23,3: 467-468. - Histoire sociale-Social History, no 1, 22,3: 449-450. - Kipping, Ernst, The Hessian View of America 1776-1783, 25,4: 581. Québec-Histoire, vol. 1 , no 1 , février-mars $1971,25,1$ : 124-125.

Blain, Jean: Cornell, Hamelin, Ouellet, Trudel, Canada-Unity in Diversity/ Canada-Unité et diversité, 22,3: 450466. - Dumas, Silvio, Les filles $d u$ Roi en Nouvelle-France. Étude historique avec répertoire biographique, n.c., 27,3: 412-414. - Eccles, William J., The Canadian Frontier, 1534-1760, n.c., 25,3: 400-407; 25,4: 608. - Frégault, Guy, Le XVIIIe siècle canadien. Études, 22,3: 518; 22,4: 683; 23,1: 122-126, 181. - Lahontan, baron de, Nouveaux voyages de $\mathrm{Mr}$ le Baron de Lahontan, n.c., 27,3: 409-410. Lanctot, Gustave, Filles de joie ou Filles du Roi, étude sur l'émigration féminine en Nouvelle-France, n.c., 27,3: 410-412. - Séguin, Robert-Lionel, La vie libertine en Nouvelle-France au XVIle siècle, n.c., 27,3: 414-416. 
Blain, Marc A., Ramos, D. et al, El Consejo de la Indias en el siglo XVI, 25,2: 264-265.

Bonenfant, Jean-Charles: Arès, Richard, s.j., Nos grandes options politiques et constitutionnelles, 26,2: 271-272.

Howe, Joseph, Opportunist? Man of Vision? Frustated Politician?, 24,3: 432-433. - LaPierre, Laurier-L., Québec: hier et aujourd'hui, 22,3: 481. Ryerson, Stanley-Bréhaut, Le capitalisme et la Confédération. Aux sources du conflit Canada-Québec (1760-1873), 28,1: 121-122. - Sabourin, Louis, Me, dir., Le système politique du Canada, 23,1: 131-133. - Schindeler, F. F., Responsible Government in Ontario, 23,2: 322 .

Bonville, Jean de: Godin, Pierre, L'information-opium - Une histoire politique du journal La Presse, 29,4: 589-591.

Boucher, Jacques: Baillargeon, Georges-E., La survivance du régime seigneurial à Montréal, 22,1: 128-131.

Bower, Peter: Rawlyk, G. A., Yankees at Louisbourg, 22,2: 313-316.
Brochu, Michel: Johnson. Alice M., éd. Saskatchewan Journals and Correspondance 1795-1802, 24,1: 96-99.

Brouillette, Benoît: Spry, Irene M., The Papers of the Palliser Expedition 1857-1860, 24,3: 440-444.

Brunet, Michel: Audet, Louis-Philippe, Bilan de la réforme scolaire au Québec, 1959-1969, 23,3: 466-467. - Canada, un siècle, 1867-1967, 22,1: 118 119. - Cuff, R. D. et J. L. Granatstein, Canadian-American Relations in Wartime: From the Great War to the Cold War, 30,3: 417-418. - Firestone, O. J., Industry and Education - $A$ Century of Canadian Development, 23,2: 303-304. - Garner, John, The Franchise and Politics in British North America, 1755-1867, 23,2: 304-306. Granatstein, J. L., The Politics of Survival: The Conservative Party of $\mathrm{Ca}$ nada, 1939-1945, 22,1: 116-118. Penlington, Norman, éd. On Canada: Essays in Honour of Frank H. Underhill, 25,2: 260-261. - Stanley, George, F. G., A short History of the Canadian Constitution, 23,3: 465-466. - Upton, L. F. S., The Loyal Whig William Smith of New York \& Québec, 23,2: 306-308.
Cadieux, Lorenzo, s.j.,: Provost, Honorius, ptre, Sainte-Marie de la Nouvelle-Beauce, 21,2: 318-319.

Campeau, Lucien, s.j.,: Baillargeon, Noël, Le Séminaire de Québec sous l'épiscopat de Mgr de Laval, 27,1: 91-93. - Beaudoin, Louis, éd., La Recherche au Canada français, 24,1: 88. - Bélanger, René, Les Basques dans l'estuaire du Saint-Laurent, 25,3: 409-410. - Dumont-Johnson, Micheline, Apôtres ou Agitateurs. La France missionnaire en Acadie, 24,3: 429-430. - Gariépy, Raymond, Les Seigneuries de Beaupré et de l'Ile d'Orléans dans leurs débuts, 29,1: 104-105. - Groulx, Lionel, Constantes de vie, 21,2: 316317. - Hamilton, Raphël N., s.j., Marquette's Explorations. The Narratives reexamined, 24,4: 605-606. -
Lacroix, Benoît, Lionel Groulx, textes choisis et présentés, 21,2: 317-318. Laflèche, Guy, Relation de 1634 de Paul Lejeune. Le missionnaire, l'apostat, le sorcier, 27,4: 593-594. - LeMoine, Roger, L'Amérique et les Poètes français de la Renaissance, 28,3: 438. - Mahn-Lot, Marianne, La découverte de l'Amérique, 25,2: 258. Maillet, Antonine, Rabelais et les traditions populaires in Acadie, 27,1: 109-110. - Ministère des Affaires culturelles, Rapport des Archives nationales du Québec, 19/3 (tome 51), 28,4: 595-596. - Morison, Samuel Eliot, The European Discovery of America The Northern Voyages A. D. 500-1600, 26,1: 113-114. - Orban, Edmond, Le Conseil législatif de Québec, 18671967, 21,3: 484-485. - Pendergast, 
James F. et G. Bruce Trigger, Cartier's Hochelaga and the Dawson Site, 27,1: 112-113. - Rapport des Archives du Québec 1966, tome 44, 21,3: 485-486. - Rapport des Archives nationales du Québec 1973, tome 51, 28,4: 595596. - Recherches sociographiques (VIII, no 2-1967), 21,3: 486. - Seary, E. R., Place Names of the Avalon Peninsula of the Island of Newfoundland, 27,1: 115-117. - Séguin, RobertLionel, La civilisation traditionnelle de "l'Habitant" aux 17 e et 18 e siècles, fonds matériel, 22,1: 114-116. Toujas, René, Le Destin extraordinaire du gascon Lamothe-Cadillac, fondateur de Détroit, 28,4: 603. - Tremblay, Victor, Le Poste de Métabetchouan, 29,2: 287-288. - Walsh, H. H., The Church in the French Era from Colonization to British Conquest, 21,4: 828-829.

Cartier, Georgette S.: Boletin de Historia y Antiquedades, 24,1: 105-107. Gonzalez, Elda R. et Mellafe, Rolando, La fonction de la famille dans l'histoire sociale des colonies hispano-américaines, 22,1: 144-149. Morazzani De Pérez Enciso, Gisela, La Intendencia en Espana y en America, 21,1: 137-141. - Pla, J., La propriété rurale en Amérique latine, 23,1: 143-150.

Castonguay, Charles: Joy, Richard J., Languages in conflict: The Canadian Experience, 28,2: 276-280.

Caya, Marcel: Armstrong, F. H. Stevenson, H. A. et Wilson, J. D., éd., $A s$ pects of Nineteenth-Century Ontario. Essays presented to James J. Talman, 28,2: 271. - Beaudin, François, compilateur, Archivistique québécoise, 29,2: 272-273. - Crunican, Paul, Priests and Politicians: Manitoba Schools and the Election of 1896, 29, 2: 276-277. - Oliver, Peter, Public \& Private Persons. The Ontario Political Culture, 1914-1934, 30,3: 426-428. Robin, Martin, Canadian Provincial Politics. The Party Systems of the Provinces, 27,1: 114-115. - St-Amant, Jean-Claude et al., Répertoire des élections fédérales dans la ville de Sudbury (1887-1972), 29,3: 439-440. Swainson, Donald, dir., Oliver Mowat's
Ontario. Papers presented to the Oliver Mowat Colloquium, 27,2: 295. - Thomas, Lewis H., The Renaissance of Canadian History: $A$ Biography of A. L. Burt, 30,1: 119-121. - Waite, Peter B., Canada, 1874-1896. Arduous Destiny, 27,3: 442-443.

Chalifoux, Jean-Pierre: Rolland-Thomas, Paule, Victor Coulombe, s.j. et Juliette Chabot, Vocabulaire technique de la bibliothéconomie et de la bibliographie suivi d'un lexique anglaisfrançais, 24,3: 439-440.

Charbonneau, Hubert: Arès, Richard, Les positions-ethniques, linguistiques et religieuses - des Canadiens français à la suite du recensement de 1971, 30,2: 270-272. - Dechêne, Louise, Habitants et marchands de Montréal au XVIIe siècle, note critique, 30-2: 263-269. - Dujấquier, Jacques, introduction à la démographie historique, 30,3: 437-438. - Trudel, Marcel, La population du Canada en 1663, n.c., 27,3: 417-424.

Chevalier, Willie: Rumilly, Robert, Histoire de la province de Québec: tome $X X X I X$ - La guerre de 1939-1945 Le plébiscite, 23,3: 486-488.

Cliche, Marie-Aimée: Trudel, Marcel, The Beginnings of New France, 15241663, 28,1: 127-129.

Comeau, Paul-André: Rumilly, Robert, Histoire de la province de Québec: tome XL, La Guerre de 1939-1945 Le Bloc Populaire, 23,3: 488-490.

Comeau, Robert: Bédard, Roger-J., éd., L'Essor économique du Québec, 23,3: 477-481. - Tremblay, Louis-Marie, Le syndicalisme québécois. Idéologies de la C.S.N. et de la F.T.Q. (19401970), 27,2: 297-301.

Cooke, Owen: Morton, Desmond et Roy, Reginald H., éd., Telegrams of the North-West Campaign, 1885, 27,2: 287-288.

Courteau, Guy, s.j.: Tremblay, Victor, prélat, Les trente aînées de nos localités - Brefs historiques - Au royaume du Saguenay, 23,2: 308-310.

Cousineau, Jacques, s.j.: McDonough, John Thomas, Charbonneau et le Chef, 28,3: 442-444.

Crowley, T. A.: Arsenault, Bona, Louisbourg, 1713-1758, 26,2: 272-274. 


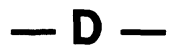

Daigle, Jean: Clark, Andrew Hill, Acadia-The Geography of Early Nova Scotia to 1760, 23,2: 328-330.

Daoust, Paul: Timlin, Mabel F. et Albert Faucher, Les sciences sociales au Canada - The Social Sciences in $\mathrm{Ca}$ nada, 23,3: 491-493.

Debien, G.: Frostin, Charles, Histoire de l'autonomisme colon de la partie française de Saint-Domingue aux XVIIe et XVIIIe siècles. Contribution à l'étude du sentiment américain d'indépendance, 28,1: 116-118.

Denault, Bernard: Archives de Sciences sociales des Religions, no 37, janvierjuin 1974, 29,2: 270-271. - Guide sommaire des Archives des communautés religieuses au Canada, Abridged Guide to the Archives of Religious Communities in Canada, 29,2: 280281.

Desbiens, Albert: Morchain, Janet, Sharing a Continent, 28,2: 281-282. - Orban, Edmond, La Présidence moderne aux États-Unis, 29,3: 435-436.

Deshaies, Bruno: Brunet, Michel, Québec-Canada anglais. Deux itinéraires, un affrontement, 22,4: 625-633.

Désilets, Andrée: Berger, Carl, Imperialism and Nationalism, 1884-1914: A Conflict in Canadian Thought, 25,3: 410-411. - Granatstein, J. L. et Paul Stevens, éd., Canada since $1867-a$ bibliographical guide, 28,3: 428-429. - Gravel, Jean-Yves, L'armée au Québec (1868-1900): un portrait social, 29,2: 280. - La Terreur, Marc, Les tribulations des conservateurs au Québec, de Bennett à Diefenbaker, 27,4: 594-596. - Lavallée, André, Québec contre Montréal. La querelle universitaire, 1876-1891, 29,2: 286-287. - Pouliot, Léon, s.j., Monseigneur Bourget et son temps - tome III: L'Évêque de Montréal - Deuxième partie: La marche en avant du diocèse, 18461876, 27,3: 438-439.

Desrosiers, Richard.-F.: Brossard, Jacques, La Cour Suprême et la Constitution - Le forum constitutionnel au Canada, 23,3: 475-477.

Dickinson, John. A.: Nish. Cameron, François-Étienne Cugnet 1719-1751: entrepreneur et entreprises en Nouvelle-France, 30,2: 280-284.

Dorge, Lionel: Roy, Marie-Anna A., La Montagne Pembina au temps des colons - Historique des paroisses de la région de la Montagne Pembina et biographie des principaux pionniers, 25,2: 266-267. - Roy, Marie-Anna A., Les Visages du vieux Saint-Boniface, 25,2: 266-267.

Dubé, Jean-Claude: Vachon, André, L'administration de la NouvelleFrance, 1627-1760/The Administration of New France 1627-1760, 25,3: 429.

Dufour, Pierre et Gérard Goyer: Archives publiques du Canada et Conseil canadien de recherches sur les humanités, Catalogue collectif des manuscrits des archives canadiennes, 29,4: 579582.

Duhamel, Roger: Ethier-Blais, Jean, Signets I et II, 21,1: 143-145. - Gay, Paul, Survol de la littérature canadienne-française, 21,4: 835-836. - Hare, John et Jean-Pierre Wallot, Les imprimés dans le Bas-Canada, 1801-1810, 22,1: 131-133. - Ménard, Jean, Xavier Marmier et le Canada. Relations franco-canadiennes au XIXe siècle, 21,2: 312-313. - Moisan, Clément, L'âge de la littérature canadienne, 23,4: 628-630.

Dumont, Fernand: Angers, FrançoisAlbert, Pour orienter nos libertés, 24,4: 597-598.

Dupont, Antonin: Bilodeau, Rosario, Robert Comeau, André Gosselin et Denise Julien, Histoire des Canadas, 26,1: 106-107. - Fournier, Rodolphe, Lieux et monuments historiques de l'île de Montréal, 29,1: 103-104. Rumilly, Robert, Histoire de la province de Québec - XLI - La guerre de 1939-1945, Duplessis reprend les rênes, 24,2: 296-298.

Durocher, René: Cornell, Hamelin, Ouellet, Trudel, Canada-Unity in Diversity/Canada-Unité et diversité, 22,3: 450-466. - Garigue, Philippe, Bibliographie du Québec (1955-1965), 21,3: 487-490. - Hamelin, Marcel, éditeur, Les Mémoires du sénateur Raoul Dandurand (1861-1942), 21,4: 
844-847. - Lebel, Gilles, Horizon 1980, Une étude sur l'évolution de l'économie du Québec de 1946 à 1968 et sur ses perspectives d'avenir, 26,1: 122. - Robert, Jean-Claude, $\mathrm{Du} \mathrm{Ca}$ nada français au Québec libre. Histoire d'un mouvement indépendantiste,
29,3: 437-439. Veilleux, Gérard, Les relations intergouvernementales au Canada, 1867-1967 - Les mécanismes de coopération, 26,1: 120-121 Ward, Norman, ed., A Party Politician. The Memoirs of Chubby Power, 21,1: 128-131.

\section{- E-}

Eccles, W.-J.: Hardy, James D. J., Judicial Politics in the Old Regime. The Parlement of Paris during the Regency, 23,4: 616-617.
Eid, Nadia F.: «L'Étude des idéologies au Québec: bilan et perspectives de la recherche», note critique, 25,4: 558564.

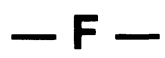

Falardeau, Jean-Charles: Bouchard, Gérard, Le village immobile. Sennelyen-Sologne au XVIIIe siècle, 27,3: 426-428. - Brunet, Jacques, Albert Laberge, sa vie et son auvre, 24,1: 89-91. - Robert, Guy, Aspects de la littérature québécoise, 25,3: 426.
Falmagne, Jacques: Sanfaçon, Roland, Défrichements, peuplement, institutions seigneuriales en Haut-Poitou du Xe au XIIIe siècle, 21,3: 495-496.

Faucher, Albert: Lavoie, Yolande, L'émigration des Canadiens aux Etats-Unis avant 1930 - Mesure du phénomène, 26,4: 596-597.

\section{$-\mathbf{G}-$}

Gagnon, François-Marc: Trudel, Jean, L'orfevrerie en Nouvelle-France, 30,2 : 284-289. - Trudel, Jean, Un chefd'ouvre de l'art ancien du Québec: la chapelle des Ursulines, 27,1: 118-119.

Gagnon, Jean-Pierre: Dawl, Edward H., Hélène Espesset, Marc LaFrance, et Thierry Ruddell, La ville de Québec, 1800-1850: un inventaire de cartes et plans, 29,4: 587-589.

Gagnon, Serge: Bernard, Jean-Paul, Les idéologies québécoises au 19e siècle, 27,4: 579-581. - Denault, Bernard et Benoît Lévesque, Éléments pour une sociologie des communautés religieuses au Québec, 30,3: 418-422. - Dictionnaire biographique du Canada -
Volume II: De 1701 à 1740, 25,2. 256-257.

Galarneau, Claude: Hare, John et JeanPierre Wallot, Confrontations-Ideas in conflict. Choix de textes sur des problèmes politiques, économiques et sociaux du Bas-Canada (1806-1810), 26,4: 594-595.

Garon, Robert: Archives publiques du Canada. Division des manuscrits, Inventaire général - Manuscrits/General Inventory-Manuscripts, 29,2: 269270.

Gay, Paul, ptre, c.s.sp.: Béchard, Henri, s.j., L'hérö̈que indienne Kateri Tekakwitha, 21,3: 490-491. - Drolet, Antonio, La Ville de Québec, histoire muni- 
cipale, III. De l'incorporation à la Confédération (1833-1867), 22,1: 127 128.

Genest, Jean-Guy: Gasgrain, Thérèse F., Une femme chez les hommes, 27,1: 96-99. - Chaloult, René, Mémoires politiques, 25,4: 568-569. - Durocher, René et Paul-André Linteau, Histoire du Québec - Bibliographie sélective (1867-1970), 26,1: 110-111. - Raynault, Adhémar, Témoin d'une époque, 26,1: 117-119.

Gervais, Gaétan: Centre de recherche en civilisation canadienne-française, Université d'Ottawa, Actes du colloque sur la situation de la recherche sur la vie française en Ontario, 30,1: 110113.

Giguère, Georges-Émile s.j.: Donnelly, Joseph P., s.j., The Journals and Paintings of Father Nicolas Point - Indian Life in the Rocky Mountains, 18401857, 22,3: 478-480. - Gariépy, Raymond, Le Village du Château-Richer (1640-1870), 23,4: 615-616. - Handlin, Oscar, éd., American Problem Studies, 22,4: 652-654. - Héroux, Denis, Robert Lahaise et Noël Vallerand, Initiation à la Nouvelle-France, 22,1: 108112. - Lemieux, Lucien, L'établissement de la première province ecclésiastique au Canada 1783-1844, 23,2: 330-332. - Saucier, Roger, Comment enseigner l'histoire, 21,1: 151-153.

Gosselin, André: Schull, Joseph, Laurier, 23,2: 319-322.

Gosselin, Paul-Émile: Courteau, Guy, s.j., Le docteur J.-Raoul Hurtubise, m.d.-m.p.-40 ans de vie française à Sudbury, 25,4: 572-573.

Gouin, Jacques: Boissonnault, Charles-Marie, Histoire politicomilitaire des Canadiens français (1763-1945), 21,4: 833-835. - Boult, Reynald, Bibliographie du droit canadien, 22,4: 636-637. - Bulletin de la Société Historique Franco-Américaine, Nouvelle série: vol. XII, 22,1: 126127. - Dagenais, Gérard, Dictionnaire des difficultés de la langue française au Canada, 22,1: 121-122. - Florentin, Eddy, Stalingrad en Normandie, 21,3: 498-499. - Kavanagh, Martin, La Vérendrye: His Life and Times, $A$ Biography and a Social Study of $a$ Folklore Figure, Soldier, Fur Trader,
Explorer, 22,2: 316-317. - Kennett, Lee, The French Armies in the Seven Years'War, 21,3: 496-498. - Morin, Renée, Un bourgeois d'une époque révolue: Victor Morin, notaire (18651960), 21,4: 831-833. - Nicholson, G.W.L., The Gunners of Canada: The History of the Royal Regiment of $\mathrm{Ca}$ nadian Artillery, 26,2: 285-287. Reed, John F., Valley Force Crucible of Victory, 24,3: 438-439. - Séguin, Robert-Lionel, La victoire de SaintDenis, 22,1: 122-124. - Smith, Samuel Stelle, Fight for The Delaware 1777, 25,2: 267-268. - Stanley, George F.G., New France: The Last Phase (1744-1760), 23,2: 314-316. - Swettenham, John, McNaughton, Vol. I: 1887-1939, 22,3: 485-487. - Swettenham, John, McNaughton, Vol. II : 1939-1943 et Vol. III: 1944-1966, 23,4: 646-648. - Vasseur, André-Georges, Boulogne 1944: Jusqu'au dernier, 23,2: 316-317. - Yuzyk, Paul, Les Canadiens-Ukrainiens: leur place et leur rôle dans la vie canadienne, 22,1: 124-126.

Gourd, Benoît-Beaudry: Benoist, Émile, L'Abitibi, pays de l'or, n.c., 30,1: 104-105. - Biays, Pierre, Les marges de l'okoumène dans l'Est du Canada, n.c., 30,1: 101. - Blanchard, Raoul, Les pays de l'Ottawa. L'AbitibiTémiscamingue, n.c., 30,1: 101. Dumas, Evelyn, Dans le sommeil de nos os. Quelques grèves au Québec de 1934 à 1944, n.c., 30,1: 105-106. Gregory, J. F., A geographical analysis of the Ontario-Quebec Metals region, n.c., 30,1: 102. - Lafleur, Normand avec la collaboration de Donat Martineau et Alice Descôteaux. La vie quotidienne des premiers colons en Abitibi-Témiscamingue, 30,3: 424-426. - Larouche, Fernand, L'immigrant dans une ville minière au Québec, n.c., 30,1: 106. - Moore, E. S., American influence in Canadian mining, n.c., 30,1: 102. - Roberts, Leslie, Noranda, n.c., 30,1: 103. - Villemure, Martien, Les villes de la Faille de Cadillac, n.c., 30,1: 101-102.

Gow, J. I.: Lajoie, André, Les Structures administratives régionales - Déconcentration et décentralisation au Québec, 23,1: 126-129. 
Goyer, Gérard et Pierre Dufour: Catalogue collectif des manuscrits des archives canadiennes, 29,4: 579-582.

Gravel, Jean-Yves: Foner, Jack D., The United States Soldier Between Two Wars: Army Life and Reforms, 18651898, 25,1: 119-121. - Fusiliers Mont-Royal, Cent ans d'histoire d'un Régiment canadien-français, les Fusiliers Mont-Royal, 1869-1969, 26,3: 433-436. - Gouin, Jacques, Par la bouche de nos canons. Histoire du 4 e régiment d'artillerie moyenne/4th $\mathrm{Cdn}$ Medium Rgt. RCA - 1941-45, 24,2: 292-293. - Granatstein, J. L., Canada's War. The Politics of the Mackenzie King Government, 19391945, 29,1: 105-107. - Laflamme, Jean, Les camps de détention au Québec durant la première guerre mondiale, 27,1: 106-107. - Morton, Desmond, Ministers and General Politics and the Canadian Militia 1868-1904, 24,4: 609-611. - Morton, Desmond, The Last War Drum. The North West Campaign of 1885, 27,1: 111-112. -
Swettenham, John, D-Day. Jour-J., 23,3: 493-494.

Grenon, Michel: Devèze, Michel, L'Europe et le Monde à la fin du XVIIIe siècle, 26,1: 107-109.

Grenon, Pierre Michel: Dhoquois, Guy, Pour l'Histoire, n.c., 28,2: 263-269. Veyne, P., Comment on écrit l'histoire: essai d'épistémologie, n.c., 28,2: 259-263, 267-269.

Grimard, Jacques: De Valk, Alphonse, c.s.b., History Collection. Canadian Catholic Church/Collection d'histoire, L'Église catholique canadienne, 29,3: 445-446.

Groulx, Lionel, ptre: Mitchell, E., Messire Pierre Boucher (écuyer) seigneur de Boucherveille 1622-1717, 21,1: 132133. - Morin, Rosaire, Réalités et perspectives économiques - Faut-il confier à New York l'avenir des Canadiens français?, 21,1: 133-134. Sœur Thérèse-du-Carmel, s.s.j. (Lucienne Blais), Bibliographie analytique de l'ouvre de Félix-Antoine Savard, 21,1: 145-146.

\section{- H -}

Hamelin, Louis-Edmond: Lamontagne, Roland, La Baie James, dans l'histoire du Canada, 28,3: 434-436.

Hardy, René: Groupe de recherche en histoire régionale, Bibliographie d'histoire des Cantons de l'Est, 30,3: 422424. - Labarrère-Paulé, André, Louis-François LaFlèche, 24,2: 294 296.

Harvey, Fernand: Bernier, Jacques, Condition ouvrière à Montréal à la fin du XIXe siècle 1774-1896, n.c., 24,4: 575-576. - Hamelin, Jean, Paul Larocque et Jacques Rouillard, Répertoire des grèves dans la province de Québec au XIXe siècle, 24,4: 604-605. - Larocque, Paul, La condition socio-économique des travailleurs de la ville de Québec (1896-1914), n.c., 24,4: 574-575. - Mathieu, Jacques-P., La condition ouvrière dans l'industrie du cuir à Québec, de 1900 à 1930, d'après les procès-verbaux d'un syndicat, n.c., 24,4: 576-577. - Revue de l'Institut de Sociologie, "Aperçu sociologique sur le Québec", 23,1: 150154. - Rouillard, Jacques, Les filatures de coton au Québec, 1900-1915, n.c., 24,4: 571-573. - Ruddell, David Terence, Apprenticeship in early nineteenth century Quebec, 1793-1815, n.c., 24,4: 568-570.

Houde, Roland: Bibliothèque nationale du Québec, Les ouvrages de référence du Québec, 23,4: 637-645.

Hould, Claudette: Nappen, Luc, Notre-Dame-de-Québec. Son architecture et son rayonnement (1647-1922), 28,4: 593-595.

Hurtubise, Pierre, o.m.i.: Cahiers du Québec, no 13, L'Hôtel-Dieu de Montréal (1642-1973), 27,3: 429-430. 


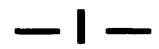

Igartua, José E.: Chabot, Richard, Le curé de campagne et la contestation locale au Québec (de 1791 aux troubles de 1837-1838), 29,4: 582-585. Comeau, Robert, éd., Économie qué- bécoise, 25,3: 415-417. - Masson, Henri, Joseph Masson, dernier seigneur de Terrebonne 1791-1847, 26,3: 441-442.
Jaenen, Cornelius J.: Campeau, Lucien, s.j., La première mission des Jésuites en Nouvelle-France (1611-1613) et Les commencements du Collège de Québec (1626-1670), 28,1: 113-114. - Dorge, Lionel, Introduction à l'étude des Franco-Manitobains. Essai historique et bibliographique, 28,1: 114-116. Trigger, Bruce G., The Huron: Farmers of the North, 25,3: 426-429.

Jetté, Denis: Desjardins, Dr Edouard, Suzanne Giroux et Eileen C. Flanagan, Histoire de la profession infirmière au Québec, 25,3: 417-418. - Déziel, Julien et collaborateurs, Médaillons d'ancêtres, 25,3: 418. - Douville, Raymond, Pierre Boucher. Textes choisis, 27,1: 101-102. - Groulx, Lionel, Roland-Michel Barrin de La Galissonière, 24,4: 602-603.

Johnson, Micheline [Dumont]: Boulet, Gilles, Jacques Lacoursière et Denis Vaugeois, Le Boréal Express, tome III: 1810-1841, 27,2: 282. - Cleverdon, Catherine, The Woman Suffrage movement in Canada, n.c., 29,3: 428. - Dragon, Antonio, L'Acadie et ses 40 Robes Noires, 28,3: 427-428. Dussault-Dumas, Huguette et Christian Laville, Initiation à l'Histoire et aux Sciences de l'homme, 29,1: 109-111. - Gagnon, Mona-Josée, Les femmes vues par le Québec des hommes, 30 ans d'histoire des idéologies, 19401970, n.c., 29,3: 427-428. - Houle, Ghislaine, La femme au Québec, n.c., 29,3: 425. - Howard, Richard, Jacques Lacoursière et Claude Bouchard,
A New History of Canada, 27,4: 590 594. - Jean, Michèle, Québécoises du $X X$ siècle, n.c., 29,3: 426. - Lacoursière, Jacques et Claude Bouchard, Notre Histoire: Québec-Canada, 27,4: 590-594. - Lavallée, Yvon et Daniel Paquette, L'Histoire aujourd'hui/hier, 29,1: 109-111. - Les Cahiers des Dix, nos 35 et 36, 27,3: 428. - Lefebvre, André et al., Initiation à l'histoire à partir du monde actuel..., 27,1: 107 109. - Stephensen, Marylee, Women in Canada, n.c., 29,3: 425-426.

Jolicœur, Catherine: Bernier, Hélène, $\mathbf{L a}$ fille aux mains coupées (conte-type 706), 25,3: 411. - Griggs, Mary-Ann, La chanson folklorique dans le milieu canadien-français traditionnel. The Folk-Song in the Traditional Society of French-Canada, 24,2: 293-294.

Jones, Richard. A.: Choquette, Robert, Language and Religion - A History of English-French Conflict in Ontario, 29,4: 585-587. - Dupont, Antonin, Les relations entre l'Eglise et l'État sous Louis-Alexandre Taschereau, 1920-1936, 27,4: 584-586. - Lacoursière, Jacques, Alarme citoyens! L'affaire Cross-Laporte - du connu à l'inconnu, 26,2: 282-283. - Milner, Sheilagh Hodgins, Henry Milner, The Decolonization of Quebec, 28,3: 445-446. - Thomson, Dale C., éd., Quebec Society and Politics: Views from the Inside, 28,4: 596-597. - Trofimenkoff, Susan Mann, Action française; French Canadian Nationalism in the Twenties, 30,1: 121-123. 


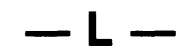

LaBrèque, Marie-Paule: Longpré, Anselme, Ephrem Longpré, 1890-1965, 28,4: 603 .

Lachance, André: Les Cahiers des Dix, no 30, 21,1: 141-143. - Ministère des Affaires culturelles, Rapport des Archives nationales du Québec, 1971, tome 49, 27,3: 436-437. - Trudel, Marcel, Les débuts du régime seigneurial au Canada, 29,3: 441-443.

Lacroix, Benoît, o.p.: Groulx, Lionel, Mémoires I, II, III, IV, n.c., 24,3: 413-419. - Jolicœur, Catherine, Le Vaisseau Fantôme. Légende étiologique, 24,4: 606-607. - Laforte, Conrad, Poétiques de la chanson traditionnelle française, 30,2: 273-275. - Schmitz, Nancy, La Miensongère iconte-type 710), 27,3: 439-440. - Trofimenkoff, Susan Mann, Abbé Groulx - Variations on a Nationalist Theme, n.c., 28,3: 415-420.

Lafrance, André: Bonneville, Léo, dir., Dossiers du Cinéma I, 23,3: 469-470.

Lahaise, Robert: Marsan, Jean-Claude, Montréal en évolution, 28,3: 439-442.

Lajeunesse, Marcel: Audet, LouisPhilippe et Armand Gauthier. Le système scolaire du Québec; organisation et fonctionnement, 22,2: 309-310. Audet, Louis-Philippe, Histoire de l'enseignement au Québec, 26,3: 426432. - Henry, Ginette, Répertoire des périodiques québécois: Ire partie, 29,1: 108-109. - Nadeau, Eugène, o.m.i., La Femme au cour attentif: Mère Gamelin, 23,3: 490-491. - Parizeau, Gérard, La société canadiennefrançaise au XIXe siècle - Essais sur le milieu, 30,1: 116-117.

Lamonde, Yvan: Arguin, Maurice, Fernand Harvey et Raymond Blais, Inventaire des projets de recherche sur le Québec à l'Université Laval 1972-1973, 27,3: 432-435. - Chalifoux, JeanPierre, Bio-bibliographies et bibliographies. Liste des travaux bibliographiques des étudiants en bibliothéconomie de l'Université de Montréal, 25,3: 413-414. - Dumont, Fernand et Pierre St-Arnaud, Initiation bibliographique à l'étude de la sociologie de la connaissance, 27,3: 432-435. - Galarneau,
Claude, La France devant l'opinion canadienne (1760-1815) 24,4: 600-602. - Harvey, Fernand et Peter Southam, Chronologie du Québec, 1940-1971, 26,1: 157; 27,3: 432-435. - Harvey, Fernand, Inventaire des cartes socioéconomiques sur le Québec, 1940-1971, 26,1: 157; 27,3: 433-435. — «Histoire, sciences humaines et culture au Québec (1955-1970)», n.c., 25,1: 106-113. - Institut sup. des sciences humaines, coll. «Instruments de travail », nos 1-8, 27,3: 432-435. - Laurin, Christiane, Périodiques canadiens sur microfilms. Liste des microfilms disponibles au Québec dans les bibliothèques universitaires et à la Bibliothèque nationale, 25,3: 422-423. - Southam, Peter, Bibliographie des bibliographies sur l'économie, la société et la culture du Québec, 1940-1971, 26,1: 157, 27,3: 432-435. - Twaites, J. D., L'enseignant québécois, sources et études récentes, 27,3: 432-435. - Vinet, Alain, Épistémologie et sociologie de la médecine: bibliographie, 27,3: 432-435.

Lamontagne, Roland: Braudel, Fernand, La Méditerranée et le monde méditerranéen à l'époque de Philippe II, 21,1: 148.

Laperrière, Guy: Beaulieu, Victor-Lévy, Manuel de la petite littérature du Québec, 29,3: 445. - Clément, Gabriel, Histoire de l'Action catholique au $\mathrm{Ca}$ nada français, 27,1: 120-124. - Harvey, Fernand, Aspects historiques du mouvement ouvrier au Québec, 28,2: 274-276. - Hurtubise, Pierre, Germain Lesage et al., Le laïc dans l'Église canadienne-française de 1830 à nos jours, 27,1: 120-124. - Longpré, Anselme, Un mouvement spirituel au Québec (1931-1962) - Un retour à l'Évangile, 30,3: 440. - Voisine, Nive et al., Histoire de l'Église catholique au Québec (1608-1970), 27,1: 120-124.

Lapierre, Richard: Beauregard, Ludger, dir., Le Canada, une interprétation géographique, 27,3: 425-426. - Hamelin, Louis-Edmond, Le Canada, 24,1: 95. - Trudel, Marcel, Atlas de la Nouvelle-FrancelAn Atlas of New France, 23,2: 299-300. 
LaTerreur, Marc: Harris, Richard Colebrook, The Seigneurial System in Early Canada: A Geographical Study, 21,3: 499-500. - Provencher, Jean, Québec sous la loi des mesures de guerre, 1918, 26,2: 287. - Wilbur, J.R.H., éd., The Bennett New Deal: Fraud or Portent, 24,4: 611-612.

Lavallée, André: «Birth of Manitoba», The Nor'-Wester, vol. 100, no 1, 25,3: 412, - Drolet, J. C., Monseigneur Dominique Racine, bâtisseur de l'église saguenéenne, 23,1: 138-140. Johnson, J. K., dir., Les lettres de Sir John A. Macdonald, 1836-1857, 26,4: 595. - Lajeunesse, Marcel, L'éducation au Québec (19-20e siècles), 26,3: 438. - Rumilly, R., Histoire de la province de Québec: Premier gouvernement Duplessis, vol. XXVII, 23,2: 298-299. - Savard, Pierre, Jules-Paul Tardivel, la France et les États-Unis (1851-1905), 21,4: 829-831. - Wilson, J. D., R. M. Stamp et L.-P. Audet, Canadian Education: A History, 24,3: 444-445.

Lavallée, Jean-Guy: D'Allaire, Micheline, L'Hôpital-Général de Québec, 1692-1764, 27,4: 581-583. - Plante, Guy, p.m.é., Le rigorisme au XVIIe siècle: Mgr de Saint-Vallier et le sacrement de pénitence (1685-1727), 28,1: 118-119.

Lavallée, Louis: Bourgeon, Jean-Louis, Les Colbert avant Colbert. Destin d'une famille marchande, 29,2: 273276. - Lebrun, François. La vie conjugale sous l'Ancien Régime, 30,2: 275, 276. - Mandrou, Robert, Louis $X I V$ en son temps - 1661-1715, n.c., 29, 1: 87-96.

Lebel, Marc: Cauchon, Michel, JeanBaptiste Roy-Audy, 1778-c1848, 26,3: 432-433. - Lamonde, Yvan, Historiographie de la philosophie au Québec (1853-1970), 29,3: 435.

Le Blanc, Alonzo: Burger, Beaudoin, L'activité théâtrale au Québec, 29,4: 577-579.

Lebrun, André: Revue historique, $\mathrm{T}$. CCXXXIX, janvier-mars 1968, 22,3: 482-485.

Lefebvre, Jean-Jacques: Roy, Christian, p.c.s., Histoire de l'Assomption 1967, 21,3: 492-493.
Le Goff, T.J.A.: Paquet, Gilles et JeanPierre Wallot, Patronage et pouvoir dans le Bas-Canada (1794-1812). Un essai d'économie historique, 27,4: 600-603.

Lemieux, Lucien: Litalien, Rolland, Le Prêtre québécois à la fin du XIXe siècle. Style de vie et spiritualité d'après Mgr L.-Z. Moreau, 25,1: 122-123.

Le Moine, Roger: Angers, Lorenzo, Chicoutimi-Poste de traite 1676-1856, 25,1: 114-115. - Lescarbot, Marc, La Deffaite des Sauvages Armouchiquois par le Sagamos Membertou et ses Alliez Sauvages, en la Nouvelle-France au mois de juillet 1607. Où se peuvent rencontrer les ruses desdits Sauvages, leurs actes funèbres, les noms de plusieurs d'entre-eux et la manière de guérir leurs blessez, 24,1: 102-103.

Lévesque, Ulric: Rumilly, Robert, Cent ans d'éducation - Le Collège NotreDame, 1869-1969, 24,1: 103-105. Thomson, Dale C., Louis St-Laurent: Canadien, 23,2: 335-337.

Levitt, Joseph: Groulx, Lionel, Mes Mémoires, tome II, 25,3: 419-420.

Limoges, Camille: Société historique de Québec, Trois siècles de médecine québécoise, 25,1: 115-117.

Linteau, Paul-André: Beaulieu, André, Jean-Charles Bonenfant et Jean Hamelin, Répertoire des publications gouvernementales du Québec de 1867 à 1964, 22,3: 470-472. - Beaulieu, André, Jean Hamelin et Benoît Bernier, Guide d'histoire du Canada, 23,3: 473-475. - Beaulieu, André et Jean Hamelin, La presse québécoise des origines à nos jours. Tome premier: 1764-1853, note critique, 28,1: 105-107. - Bouchard, Léonard, Saint-Ferréoldes-Neiges, son historique - Parc et Station touristique du Mont Ste-Anne, 25,4: 580. - Charland-Rajotte, Ernestine, Drummondville, 150 ans de vie quotidienne au caur du Québec, 28,2: 284-285. - Comité de rénovation et de mise en valeur du Vieux-Québec, Concept général de réaménagement $d u$ Vieux-Québec, 25,2: 259-260. - Cotnam, Jacques, Contemporary Quebec: an analytical bibliography, note critique, 28,1: 108. - Desjardins, Micheline, Québec - Initiation à la géographie urbaine, 25,2: 259-260. — Dun- 
bar, Nancy J., comp., Images of Sport in Early Canada/Images du sport dans le Canada d'autrefois, 30,3: 439. Ellison, Lorraine, Peter E. Craig et William F. E. Morley, Catalogue of Canadian Newspapers in the Douglas Library, Queen's University, 24,1: 9192. - Gourd, Benoit-Beaudry, Bibliographie de l'Abitibi-Témiscamingue, n.c., 28,1: 110-111. - Hamelin, Jean et Yves Roby, Histoire économique $d u$ Québec, 1851-1896, 26,4: 590-594. Hamelin, Marcel, Les premières années du parlementarisme québécois (1867-1878), 29,2: 281-283. - Hann, R. G., G. S. Kealey, L. Kealy et P. Marriam, Primary Sources in Canadian working class history, 1860-1930, n.c., 28,1: 110. - Histoire $d u$ commerce canadien-français de Mon-

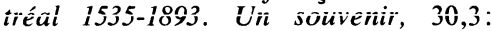
438-439. - Hughes, Everett C., Rencontre de deux mondes. La crise d'industrialisation du Canada français, 28,2: 285. - Labour/Le Travailleur, 30,3: 439-440. - Leblanc, André, E. et J. D. Thwaites, Le monde ouvrier au Québec, Bibliographie rétrospective, n.c., 28,1: 109-110. - Les Cahiers des Dix, 34, 25,2: 263-264. - Letarte, Jacques, Atlas d'histoire économique et sociale du Québec 1851-1901, 26,4: 591-594. - Limoges, José.-C., ÎleJésus. Historique et personnalités, 25,2: 259-260. - Martineau, Donat,
Le Fort Timiskaming, 25,2: 259-260. - Nish, Cameron, Le Canada français 1867-1966, Bibliographie, 22,2: 312313. - Ouellet, Fernand, Histoire économique et sociale du Québec, 1760-1850, I et II (réédition), 25,4: 581. - Provost, Honorius, SainteMarie de la Nouvelle-Beauce. II-Histoire civile, 24,3: 437-438. - Québec, Ministère de l'Industrie et du Commerce, Bureau de la statistique du Québec. Annuaire du Québec, 1971/Quebec Yearbook 1971, 25,2: 263-264. - Québec, Ministère de l'Industrie et du Commerce. Annuaire du Québecl Quebec Yearbook 1972, 26,3: 442-443. - Québec, Ministère des Affaires culturelles, Rapport des Archives nationales du Québec, 25,2: 263-264. Recherches sociographiques, IX, 1-2 (jânìièi-âû̂̂t 1968), 22.4: 6́56́-6́58. Rumilly, Robert, Histoire de la province de Québec - I-Georges-Etienne Cartier (réédition), 25,4: 582. - Savard, Pierre, éd., Paysans et ouvriers québécois d'autrefois, 23,2: 324-325. - Séguin, Georges-F., Toponymie, 25,2: 259-260. - Thibault, Claude, Bibliographie Canadiana, n.c., 28,1: 107-108. - Tremblay, Victor, Alma au Lac Saint-Jean. Son histoire, 22,2: 310-312.

Loncol, Jean-Marie: El tratado de Tordesillas y su proyeccion, 28,2: 273-274.

\section{$-\mathbf{M}-$}

Magro, René Dal: Migué, Jean-Luc, Le Québec d'aujourd'hui - regards d'universitaires, 25,4: 569-571.

Maheu, Louis: Copp, Terry, The Anatomy of Poverty - The Condition of the Working Class in Montreal, 18971929, 28,3: 423-435. - Rouillard, Jacques. Les travailleurs du coton au Québec, 1900-1915, 28,3: 446-449.

Mailhot, Raymond: Doucet, CamilleAntonio, Une étoile s'est levée en Acadie - Marcel-François Richard, 27,4: 583-584.
Maistre, Gilbert: Ministère des Terres et Forêts du Québec, Répertoire géographique du Québec, 23,3: 472.

Mann [Robertson], Susan: Roy, JeanLouis, Maîtres chez nous, 22,3: 477478.

Massicotte, Guy: Verdon, Michel, $A n$ thropologie de la colonisation au Québec. Le dilemme d'un village du LacSaint-Jean, 28,4: 598-601.

Mathieu, Jacques: Centre de Recherches sur l'histoire de la France atlantique. Enquêtes et documents-I, 26,2: 276278. - Laberge, Lionel, Rouen et le 
commerce du Canada de 1650 à 1670, 26,3: 437-438.

Mayrand, Pierre: Dyonnet, Edmond, Mémoires d'un artiste canadien, 23,3: 495-496. - Gill, Charles, Correspondance, 23,3: 494-495.

McRoberts, Kenneth: Desrosiers, Richard, éd., Le personnel politique québécois, 26,4: 586-588. - Lemieux, Vincent, Le Quotient politique vrai. Le vote provincial et fédéral au Québec, 30,2: 277-280.

Michel, Louis: Dechêne, Louise, $\mathrm{Habi}$ tants et marchands de Montréal au XVIIe siècle, n.c., 29,2: 255-268. Kurgan, G. et Ph. Moureaux, éd., La Quantification en histoire, 28,3: 430434. - LeRoy Ladurie, Emmanuel, Le Territoire de l'historien, n.c., 28,1: 95-103.

Miller, Carman: Murrow, Cassey, Henri Bourassa and French-Canadian $\mathrm{Na}$ tionalism Opposition to Empire, 23,4: 635-637. - Rawlyk, George A. et Ruth Hafter, Acadian Education in Nova Scotia-An Historical Survey to 1965 , 26,1: 115-117.
Monet, Jacques, s.j.: Berger, Carl, The Sense of Power, 26,2: 274-276. Morton, W. L., The Critical Years. The Union of British North America, 1857-1873, 25,1: 123-124.

Moquin, Michelle: De Grandpré, Pierre, Histoire de la littérature française du Québec, tome I, 22,1: 119-121. - Sylvestre, Guy et Gordon H. Green, Un siècle de littérature canadienne/A Century of Canadian Literature, 21,3: 493-495.

Morin, Claude: Institut panaméricain de géographie et d'histoire - Commission d'histoire. Colloque sur les institutions coloniales dans les Amériques au XVIII e siècle/Seminar on Colonial Institutions in the Americas of the 18th Century, Mexico, 28,4: 592-593. - Lafaye, Jacques, Quetzalcóatl et Guadalupe: la formation de la conscience nationale au Mexique (1531-1813), 29,2: 284-285.

Morton, W. L.: Clark, Lovell, The Manitoba School Question: Majority Rule or Political Rights, 25,3: 414-415.

\section{$-\mathbf{N}-$}

Nish, Cameron: Eccles, W. J., La Société canadienne sous le régime français - Canadian Society during the French Regime, 22,2: 317-319. Rish, E. E., The Fur Trade and the Northwest to 1857, 23,1: 137-138.
Nish. Élizabeth: Vaugeois, Denis. Les Juifs et la Nouvelle-France, 23,1: 129-131.
O'Neill, Louis: Grand'Maison, Jacques, Nationalisme et religion. Tome I: $N a$ tionalisme et révolution culturelle. Tome II: Religion et idéologies politiques, 24,3: 430-432.

Orban, Edmond: Caloren, Fred, Julien Harvey, Claude Julien, André Naud, Bertrand Rioux, Guy Rocher, Claude Ryan et Livia Thur, Le nouveau défi des valeurs, 23,2: 317-319. - Hepburn, James, L'Amérique brûle, 23,4: 617-618.

Ouellet, Fernand: Séguin, Maurice, L'idée d'indépendance au Québec: genèse et historique, 22,4: 637-643. Vallée, Jacques, éd., Tocqueville au Bas-Canada, 28,1: 129-132. 


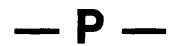

Paillé, Michel, P.: Charbonneau, Hubert, éd., La Population du Québec: études rétrospectives, 30,1: 113-115. - Lamontagne, Roland, Problématique des civilisations, 23,2: 312-314. - Larouche, Léonidas, Le Second Registre de Tadoussac, 1668-1700 - transcription, 26,3: 439-441.

Painchaud, Robert: Lupul, Manoly R. The Roman Catholic Church and the North-West School Question: a study in Church - State relations in Western Canada, 1875-1905, 29,1: 112-113.

Piérard, A.: Charbonneau, Hubert, Vie et mort de nos ancêtres - Etude démographique, 30,3: 435-436.

Poulin, Joseph-Claude: Lacroix, Benoît, o.p., L'historien au Moyen Âge. 25.3: 421-422.

Pouliot, s.j.: Bulletin de la Société Historique Franco-Américaine, nouvelle série, vol. XIII, 1967, 23,1: 142-143. - Campeau, Lucien, s.j., Monumenta Novae Franciae I, La première mission d'Acadie (1602-1616), 21,1: 125128. - Carrière, Gaston, o.m.i., His- toire documentaire de la Congrégation des Missionnaires Oblats de MarieImmaculée dans l'Est du Canada, 2e partie, Dans la seconde moitié du XIXe siècle (1861-1900), 23,2: 310-312. - Dalton, Roy C., The Jesuits' Estates Question, 1760-1880. A Study of the Background for the Agitation of 1889, 23,3: 484-486. - Desjardins, Georges, s.j., Antoine Roy dit Desjardins (1635-1684) et ses descendants, 25,4: 573-574. - Maurault, Olivier, p.a., p.s.s. Le Collège de Montréal, 17671967, 21,4: 825-826. - Mitchell, Estelle, Le soleil brille à minuit, 25,3: 423-424. - Plante, Hermann, abbé, L'Église catholique au Canada (1604 à 1886), n.c., 24,4: 581-595. - Rapport des Archives du Québec, 1967 (tome 45), 23,1: 140-142.

Provencher, Jean: Lafleur, Normand, $L a$ vie traditionnelle du coureur de bois aux XIXe et XXe siècles, 28,4: 601-602. - Patenaude, Luce, Le Labrador à l'heure de la contestation, 28,2: 292293.

\section{- R -}

Racicot, Paul-Émile, s.j.: Cercle juif de langue française, Juifs et Canadiens, 21,4: 837. - Majerus, Yvette, Le journal du Père Dominique du Ranquet, $s . j ., 21,4: 836-837$.

Rémillard, Juliette: Bibliographie annuelle de l'Histoire de France du cinquième siècle à 1945 - Année 1966, 21,3: 500-501. - Caron, abbé Adrien, Les Monts Notre-Dame, 21,4: 849-850. - Chalifoux, Jean-Pierre, Liste de sources bibliographiques relatives à la littérature canadienne-française, 21,3: 500-502. - Dictionnaire Beauchemin canadien, 22,3: 488. - Hamel, Réginald, Cahiers bibliographiques des lettres québécoises, 21,3: 500-501. - Lefebvre, Jean-Jacques, Deux siècles après Mgr Tanguay, 22,3: 487-488. Mayrand, Pierre, Sources de l'art en Nouvelle-France, 21,4: 850. - Mes fiches. Histoire du Canada, 22,3: 488489. - Ministère des Terres et Forêts. Guide toponymique du Québec-1968, 24,1: 107-108. - Nadeau, Charles, c.s.c., Saint-Joseph dans l'édition canadienne, 21,3: 500-501.

Richer, Julia: Gauthier-Landreville, Marie-Anne, c.n.d., L'île-Saint-PaulÎle-des-Sœurs près de Montréal, 24,2: 290-291.

Ricour, Françoise: Cahiers de Géographie du Québec, no 23, 22,1: 137-141. - Revue de Géographie de Montréal, vol. XXI, nos 1 et 2, 1967, 23,3: 496501 .

Robertson, Susan Mann: Roy, JeanLouis, Maîtres chez nous, 22,3: 477478.

Roby, Yves: Durocher, René et PaulAndré Linteau, Le «retard» du Qué- 
bec et l'infériorité économique des Canadiens français, 27,1: 102-103.

Rouillard, Jacques: Bobcock, Robert H., Gompers in Canada: a Study in American Continentalism before the First World War, 29,1: 99-101. - Cross, Michael S., éd. The Workingman in the Nineteenth Century, 28,4: 590-591. - De Bonville, Jean, Jean-Baptiste Gagnepetit, Les travailleurs montréalais à la fin du XIXe siècle, 30,1: 109110. - Penner, Norman, éd., Winnipeg 1919: The Strikers' own History of the Winnipeg General Strike, 27,3: 437-438. - Williams, Jack, The Story of Unions in Canada, 30,3: 431, 432.
Rousseau, Jacques: Brochu, Michel, "Étude comparative de l'évolution de la vie économique et sociale du Nouveau-Québec esquimau et indien, L'Actualité économique (1966), 21,2: 319-327.

Rousseau, Louis: Carrière, Gaston, o.m.i., Histoire documentaire de la Congrégation des Missionnaires Oblats de Marie-Immaculée dans l'Est du Canada - 2e partie - Dans la seconde moitié du XIXe siècle (18611900), 25,3: 412-413.

Rule, John C.: Filion, Maurice, Maurepas, ministre de Louis XV (1715-1749), 22,1: 101-104.
Sabourin, Louis: Morin, Claude, Le Pouvoir québécois... en négociation, 27,2: 285-287.

Saint-Germain, Yves: Bliss, Michael, $A$ Living Profit: Studies in the Social History of Canadian Business, 18831911, 28,3: 421-423. - Cuff, Robert et Glenn Porter, éd., Enterprise and $\mathrm{Na}$ tional Development: Essays in Canadian Business and Economic History, 28,3: 426-427. - Trudel, Pierre, Le Traité de réciprocité 1854, 23,2: 332333.

Salagnac, Cerbelaud: Meurgey de Tupigny, Jacques, Armorial de la Généralité de Paris, 23,4: 626-628.

Savard, Pierre: Arès, Richard, L'Église et les projets d'avenir du peuple canadien-français, 28,2: 282-283. Ayotte, Alfred et Victor Tremblay, L'aventure Louis Hémon, 28,2: 283. - Beaulieu, André et William F. E. Morley, La Province de Québec, 25,3: 408. - Blais, Jacques, De l'Ordre et de l'Aventure. La poésie au Québec de 1934 à 1944, 29,1: 114. - Boucher de Boucherville, Georges, Une de perdue, deux de trouvées, 28,2: 283. - Bruchési, Jean, Souvenirs à vaincre. Tome I, 28,4: 601. - Cadieux, Lorenzo, éd., Lettres des nouvelles missions du $\mathrm{Ca}$ nada, 1843-1852, 28,2: 280-281. - Cahiers des dix, no 31, 21,2: 313-316. Cahiers du Centre culturel canadien, no 3 , Les relations entre la France et le Canada au XIXe siècle, 29,1: 111112. - Carrière, Gaston, o.m.i., Histoire documentaire de la Congrégation des Missionnaires Oblats de MarieImmaculée dans l'Est du Canada - 2e partie - Dans la seconde moitié du $X I X e$ siècle (1861-1900), tome X, 27,1: 95-96. - Comité français des sciences historiques, Bibliographie annuelle de l'histoire de France du cinquième siècle à 1945. Année 1969, 25,1: 115. Département des littératures de l'Université Laval, sous la direction de Clément Moisan, Livres et auteurs québécois 1974-1975. Revue critique de l'année littéraire, 30,3: 436-437. Desbiens, Jean-Paul, Dossier Untel, 28,2: 284. - En collaboration, L'histoire religieuse de la France 19e et 20e siècles. Problèmes et méthodes, 29,4: 600. - Falardeau, Jean-Charles et Philippe Garigue, Léon Gérin et l'habitant de Saint-Justin, 23,4: 614-615. - Gingras, Henri, i.c. (Guy Laviolette), 1541-1974, Cap-Rouge, 30,2: 289; 30,3: 438. - Guiral, Pierre, René Pillorget et Maurice Angulhon, Guide de l'étudiant en histoire moderne et contemporaine, 25,4: 574-575. Hare, John, éd., Contes et nouvelles du Canada français, 1778-1859, 27,2: 282-285. - Joubert, Rodolphe, Édouard Montpetit - raconté par lui-même et dépeint par ses contempo- 
rains au fil de la chronique du temps, 29,4: 600-601. - Lacoursière, Jacques, Hélène-Andrée Huguet avec la collaboration de Francine Béliveau, Québec 72-73. Bilan, 29,1: 114 . Lamonde, Yvan, Louis-Adolphe $\mathrm{Pa}$ quet, 27,1: 117-118. - Lemieux, Germain, Les Vieux m'ont conté. Tome I et Tome II, 28,4: 602. - Lemire, Maurice, Les grands thèmes nationalistes du roman historique canadienfrançais, 27,2: 283-285. - Le Moine, Roger, Napoléon Bourassa, 27,1: 117-118. - Le Pluralisme, Symposium interdisciplinaire. Pluralism: its meaning to day. Travaux publiés par les soins d'Irénée Beaubien, Charles Davis, Gilles Langevin, et Robert Lapointe, 29,1: 115. - Moir, John S. $A$ History to the Christian Church in Canada. vol. II: The Church in the British Era from the British Conquest to Confederation, 27,1: 110-111. - $\mathrm{Pa}$ rizeau, Gérard, Joies et deuils d'une famille bourgeoise, 1867-1961, tome I: Notre père et nous. Tome II: Nos enfants et nous, 28,2: 284. - Poulat, Émile, Intégrisme et catholicisme intégral. Un réseau secret international antimoderniste: "La Sapinière" (19091921), 23,3: 470-472. - Rousseau, Guildo, éd., Préfaces des romans québécois du XIXe siècle, 27,2: 282-285. - Tardivel, Jules-Paul, Pour la Patrie, For my Country, An 1895 Religious and Separatist Vision of Quebec in the Mid-Twentieth Century, Int. A. I. Silver, 30,3: 440-441. - Tardivel, Jules-Paul, Pour la Patrie, roman du $X X e$ siècle, 30,3: 440-441. - Tessier, Albert, Souvenirs en vrac, 30,3: 441442. - Tremblay, Jean-Paul, Napoléon Aubin, 27,1: 117-118.

Savoie, Pierre: Allard, Michel, Jacques Archambault, Gérard Lachance, André Lefebvre et Bernard Lefebvre, La formation de l'enseignant d'histoire recherche d'une pédagogie, 22,3: 474-476.

Schmitz, Nancy: Lemieux, Germain, Placide-Eustache - Sources et parallèle du conte-type 938, 24,4: 607-609.

Séguin, Normand: Asselin, Maurice, Laurent Deshaies, Benoît-Beaudry Gourd et Fernand Larouche, AbitibiTémiscamingue, quatre études sur le Nord-Ouest québécois, 29,3: 429-431.
- Roy, Jean-Baptiste, Histoire de la corporation des agronomes de la province de Québec, 1937-1970, 27,4: 603-604.

Séguin, Robert-Lionel: Douville, R. et J.-D. Casanova, La vie quotidienne des Indiens du Canada à l'époque de la colonisation française, 21,4: 838840. - Gauthier-Larouche, Georges, L'évolution de la maison rurale laurentienne, 21,3: 491-492. - La victoire de Saint-Denis, réponse à une critique, 22,2: 319-320. - Les troubles de 1837-1838, 23,3: 473. - La Revue Imperial Oil, "Canada 1812-1871 - Période de formation », 21,2: 327-328.

Simard, Sylvain: Yon, Armand, Le Canada français $v u$ de France (18301914), 30,3: 432-435.

Smith, Donald. B.: Graymont, Barbara, The iroquois in the American Revoiution, 26,2: 281-282. - Savoie, Donat, éd., Les Amérindiens du Nord-Ouest canadien au 19 e siècle selon Émile Petitot. Vol. I: Les Esquimaux Tchiglit. Vol. II : Les Indiens Loucheux précédé d'une présentation générale des Indiens Dénè-dindjié, 25,4: 579-580.

Smoje, D.: Garneau, Saint-Denys, Oeuvres, 25,2: 257-258.

Southam, Peter: Gagnon, Marcel-Aimé, Olivar Asselin toujours vivant, 29,3: 432-434.

Stanley, George F. G.: Bowsfield, Hartwell, éd., Louis Riel: Rebel of the Western Frontier or Victim of Politics and Prejudice?, 24,4: 599-600. - Fergusson, Charles Bruce, éd., The Diary of Simeon Perkins, 1797-1803, 24,1: 92-93.

Sutto, Claude: Association canadienne des éducateurs de langue française, Esquisses du Canada français, 21,4: 827-828. - Galarneau, Claude et Elzéar Lavoie, éd., France et Canada français du XVIe au XXe siècle, 21,1: 135-137. - Hamelin, Jean et Louise Beaudoin, Les cabinets provinciaux, 1867-1967, 22,1: 141-143. - Huppert, George, The Idea of Perfect History Historical Erudition and Historical Philosophy in Renaissance, 24,3: 433435. - Recherches sociographiques, vol. 8, no 3, 22,1: 141-143. - Savard, Pierre, La vie du clergé québécois au XIXe siècle, 22,1: 141-143. - Sylvain, 
Philippe, Quelques aspects de l'antagonisme libéral-ultramontain au $\mathrm{Ca}$ nada français, 22,1: 141-143.

Sylvain, Philippe: Bernard, Jean-Paul, Les Rouges: libéralisme, nationalisme et anticléricalisme au milieu du XIXe siècle, 25.4: 565-568. - Roy, Jean-
Louis, Édouard-Raymond Fäbre, libraire et patriote canadien (1799-1854) - Contre l'isolement et la sujétion, 30,1: 117-119. - Thibault, Pierre, $S a$ voir et pouvoir. Philosophie thomiste et politique cléricale au XIXe siècle, 28,1: 124-127.

\section{- T -}

Tessier, Albert: Barbeau, Marius, Le Saguenay légendaire, 21,2: 328-329.

Thériault, Léon: Hautecœur, Jean-Paul, L'Acadie du discours. Pour une sociologie de la culture acadienne, 29,4: 591-595.

Thibault, H.-Paul: Nish, Cameron, «Le Canada français, origine à 1763 », dans Bibliographie pour servir à l'étude de l'histoire du Canada français, 22,4: 655-656.

Tousignant, Pierre: Allain, Mathé et Glenn R. Conrad, éd., France and North America: The Revolutionary Experience. Proceedings of the Second Symposium of French-American Studies, March 26-30, 1973, 28,4: 585-590. - De Lagrave, Jean-Paul, Les origines de la presse au Québec (1760-1791), 29,2: 277-279. - Graham, Gerald. S., Tides of Empire. Discursions on the Expansion of Britain Overseas, 27,3: 430-432. - Henderson, J.L.H., John Strachan, 1778-1867, 25,1: 121-122. Merk, Frederick, avec la collaboration de Lois Bannister Merk, Fruits of Propaganda in the Tyler Administration, 25,4: 577-579. - Roy, Raoul,
Résistance indépendantiste, 1793-1798. Notes pour servir à l'histoire du Franc-Canada, 27,2: 293-294. - Stanley, George F.G., Canada Invaded, 1775-1776, 28,1: 122-123.

Trigger, B.G.: "À propos d'Hochelaga", note critique, 27,2: 281.

Trofimenkoff, Susan Mann: Bélanger, André-J., L'Apolitisme des idéologies québécoises. Le grand tournant de 1934-1936, 29,1: 101-103.

Trudel, Marcel: Assiniwi, Bernard, Histoire des Indiens du Haut et du BasCanada. Tome I - Moeurs et coutumes des Algonkins et des Iroquois, 29,1: 97-99. - Tome II - Deux siècles de civilisation blanche, 1497-1685, 29,1: 97-99. - Tome III - De l'épopée à l'intégration, 1685 à nos jours, 29,1: 97-99. - Lexique des noms indiens en Amérique. Tome 1: Noms géographiques, 29,1: 97-99. - Tome II: Personnages historiques, 29,1: 9799. - Eccles, W. J., France in America, 27,1: 103-104. - Le Tenneur, René, Les Normands et les origines du Canada français, 27,4: 599-600.

\section{$-\mathbf{V}-$}

Valaskakis, Kimon: Munro, John M., Trade Liberalization and Transportation in International Trade, 23,4: 632635. - Singer, Jacques, Trade Liberalization and the Canadian Steal Industry, 23,4: 632-635.

Valois, Jacques: Desrosiers, Léo-Paul, Paul de Chomedey, sieur de Maisonneuve, 21,4: 837-838.
Vaugeois, Denis: Bowsfield, Hartwell, Louis Riel: Rebel of the Western Frontier or Victim of Politics and Prejudice?, 30,3: 428. - Bowsfield, Hartwell, Louis Riel, le patriote rebelle, 30,3: 429. - Brunet, Michel, Les Canadiens après la conquête 1759-1775 De la révolution canadienne à la révolution américaine, 24,3: 420-427. - 
Cahiers de l'Institut d'histoire, no 10 Lettres à Pierre Margry de 1844 à 1886, 23,1: 133-137. - Careless, J.M.S., The Union of the Canadas. The Growth of Canadian Institution (1841-1857), 23,2: 301-302. - Charlebois, Peter, The Life of Louis Riel, 30,3: 430. - Cormier, L.-P., prés., Lettres à Pierre Margry de 1844 à 1886, 23,1: 133-137. - Ferron, Jacques, Historiettes, 23,2: 302-303. Garneau, François-Xavier, Voyage en Angleterre et en France dans les années 1831, 1832 et 1833, 24,1: 93-95. - Howard, Joseph, Strange Empire. Louis Riel and the Metis People, 30,3: 429. - Jordon, Mary V., To Louis from your sister who loves you - Sara Riel, 30,3: 429. - Mayrand, Pierre et John Bland, Trois siècles d'architectüre aui CañuádalThiré Centuries of Ar- chitecture in Canada, 26,2: 283-285. Neatby, Hilda, The Quebec Act: Protest and Policy, 26,4: 597-598. Queen v. Louis Riel, The, 30,3: 428. - Salagnac, Georges Cerbelaud, La révolte des Métis - Louis Riel, héros ou rebelle, 30,3: 430-431. - Stanley, G.F.G., Manitoba 1870: une réalisation métisse/Manitoba 1870: A Metis Achievement, 27,3: 440-441: 30,3: 429.

Vézina, Raymond: Corbeil, Wilfrid, c.s.v., Le musée d'art de Joliette, 25,4: 571-572.

Voisine, Nive: Désilets, Andrée, Hector-Louis Langevin, un père de la Confédération canadienne (1826-1906), 26,2: 278-279. - Laperrière, Guy, $L a$ «Séparation» à Lyon (1904-1908). Étude d'opinion publique, 28,3: 436437.
Wallot, Jean-Pierre: Bonenfant, JeanCharles, La Naissance de la Confédération, 25,2: 252-254. - Cornell, Hamelin, Ouellet, Trudel, Canada-Unity in Diversity/Canada-Unité et diversité, 22,3: 450-466. - Cowan, Helen I., British Immigration Before Confederation, 25,2: 254-255: Crouzet, François, Bordeaux au XVIIIe siècle (Extrait du tome $\mathrm{V}$ de l'Histoire de Bordeaux), 22,4: 650-652. - Firth, Edith G., The Town of York, 1815-1834. A Further

\section{$-Y-$}

Young, Brian J.: Berton, Pierre. The National Dream: The Great Railway, 1871-1881, 26,4: 583-586. - Berton, Pierre, The Last Spike: The Great Railway, 1881-1885, 26,4: 583-586.
Collection of Documents of Early Toronto, 21,4: 840-844.

Watelet, Hubert: "Connaissance et sociologie de la connaissance chez les historiens». (À propos de l'article de Serge Gagnon, "La nature et le rôle de l'historiographie»), n.c., 27,4: 571-578.

Weilbrenner, Bernard: Lebel, Marc, Pierre Savard et Raymond Vézina, $A s$ pects de l'enseignement au Petit Séminaire de Québec (1765-1945), 23,2: 322-324.

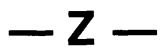

Zoltvany, Yves.-F.: Lamontagne, Roland, Textiles et documents Maurepas, 25,4: $575-576$. 